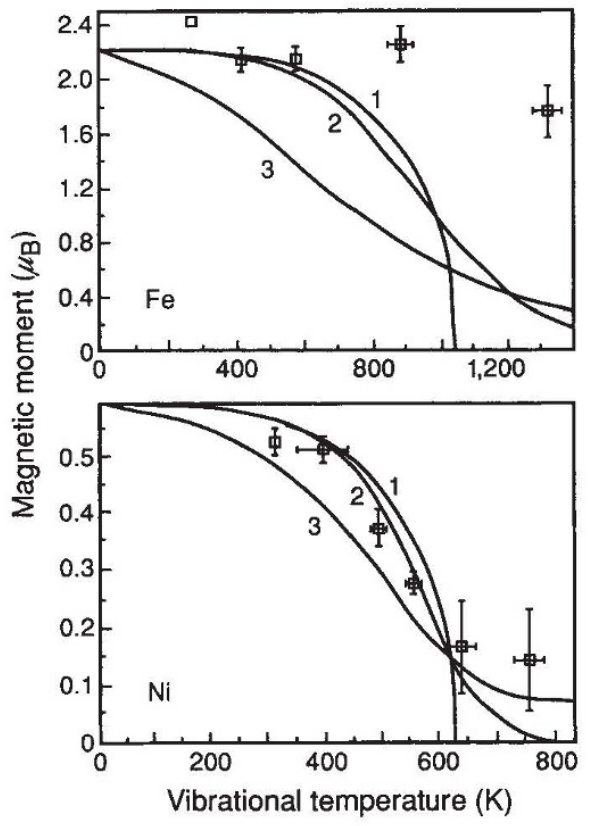

FIG. 2 Magnetic moments per atom (in Bohr magnetons, $\mu_{\mathrm{B}}$ for iron clusters with 120140 atoms and nickel clusters with $520-$ 600 atoms as a function of cluster temperature. Curves 1 are derived from temperaturedependent data for the two metals in the bulk; curves 2 use the bulk values, but take the heating of the clusters into account; curves 3 are based on the Heisenberg model as applied to clusters.

Stern and Gerlach passed a beam of sodium atoms along the axis between two long magnets, one knife-shaped, the other flat so that the magnetic field between them diverged. The sodium atoms have one valence electron so that their magnetic moment is that of the single electron. The experiment showed that half of the atoms in the beam, those with their dipoles aligned parallel to the field, are drawn towards the knife-shaped magnet (into the higher field), and those with the opposite alignment were pushed away. It was the occurrence of only two paths that revealed the quantization of spin; the degree of the deflection revealed the strength of the dipole.

In Becker and de Heer's experiments, Stern and Gerlach's technique is extended to study clusters of iron and nickel, with the expectation of observing deflections due to the presence of coupled, unpaired spin systems in the form of microscopic ferromagnets. The clusters were made by evaporating metal samples with high-powered laser pulses, and entraining and cooling the vapour in a stream of helium, in which the clusters formed by condensation (Fig. 1). Gas and clusters were then forced through a supersonic expansion, in which there is further cooling, and passed down the magnet axis. The idea was to find the cluster magnetization, $M_{z}=N \mu^{2} H / 3 k T$ ( $\mu$ is the intrinsic dipole of each atom, $H$ the applied field and $k$ Boltzmann's constant) as a function of cluster size $(N$, number of atoms) and temperature $T$. The deflection of the clusters was determined by ionizing them with a second laser, which could be scanned across the face of the magnets, and recording their arrivals in a mass spectrometer. In contrast with the original single-atom experiment, the cluster experiment gives an asymmetric distribution of deflections, towards high fields, an effect attributed $^{1}$ to the way coupling between the atoms allows them to exchange energy and angular momentum, something not possible with an isolated atom.

The magnetization could be determined from the beam deflection. The cluster temperature was derived from the rate at which atoms evaporated from the cluster following heating by yet another laser, using a formulation developed by Klots five years ago (see my previous News and Views article ${ }^{3}$. In this way, Becker and de Heer could compare the magnetization of the clusters with that of bulk materials (Fig. 2). At low temperatures, the cluster magnetic moments are close to those found for each of the bulk metals: for iron, $\mu_{\text {bulk }}=2.2$ Bohr magnetons; for nickel, $\mu_{\text {bulk }}=0.6$ Bohr magnetons. As $T$ increases towards the Curie temperature, the magnetic moment of a bulk metal rapidly approaches zero; similar behaviour is seen in the nickel clusters, but not for those of iron. It is clear from the results that the behaviour of the latter follows neither the Heisenberg model nor the temperature dependence of a bulk ferromagnet.

Becker and de Heer are not the only group to be active in this area ${ }^{4,5}$ and, although their analysis of Stern and Gerlach's experiments on small metal clusters seems to be the most comprehensive to date, there are still a number of unresolved problems in the interpretation. Experiments on metal clusters offer opportunities for understanding magnetic behaviour at an atomic level; however, the experiments could also help to identify new ferromagnetic materials. An example here might be manganese; with the metal in the form of small clusters, lattice relaxation and/ or a change in lattice structure could increase the interatomic coupling to a point where, over a narrow size range, the clusters become ferromagnetic.

Tony Stace is in the School of Chemistry and Molecular Sciences, University of Sussex, Falmer, Brighton BN1 9QJ, UK.

\footnotetext{
1. Becker, J. A. \& de Heer, W. A. Ber, Bunsenges. Phys. Chem 30, 1237-1243 (1992)

Blakemore, J. S. Solid State Physics (Cambridge Blakemore, J. S. Solid State
University Press, 1985).

3. Stace, T. Nature 327, 186-187 (1987),

4. Cox, D. M. et al. Phys. Rev. B32, 7290-7298 (1985)

5. Bucher, J. P. et al. Phys. Rev. Lett. 66, 3052 (1991)
}

DAEDALUS -

\section{The iron horse}

AGRICULTURE is, theoretically, a way of capturing the Sun's energy for human use by photosynthesis. Modern 'efficlent' agriculture in fact runs at a huge loss. The energy used to make fertilizers, drive tractors, process and pack the products and get them to market, far exceeds the solar energy thus captured. Even ecologically correct schemes like converting corn into fuelalcohol, or rape oll into diesel fuel, usually run at an energetic loss. They are simply an expensive massage for the industrial conscience.

So Daedalus has been dreaming up a genuinely energy-efficient agriculture. He is devising a diesel engine that runs directly on plant material and returns its waste to the soil, as a horse does. He recalls that Rudolf Diesel's original engine was designed to burn coal dust. DREADCO engineers are now developing a diesel to burn powdered leaves, cornstalks, rice-hulls and so on.

The easiest way to pulverize biological materials is to embrittle them by cooling with liquid air and to grind them up. All internal combustion engines sacrifice some of their power to drive a cooling system; the DREADCO cryodiesel uses a significant fraction of its power in this way, to operate its on-board cryogenic plant. The resulting flow of cryogenically cold powder is squirted directly into its cylinders.

Very fine powders burn extremely efflciently. In any case, poor burning can be neatly overcome with the aid of liquid oxygen generated by the cryogenic system. The resulting exhaust will contain a lot of solid ash, but with luck this will be so finely divided that it will polish the working parts of the engine, rather than abrading them. As a final neat touch, the waste cold from the cryogenics will be used to cool the engine's exhaust gas to ambient temperatures. The steam of combustion will condense to water, In which the alkaline plant ash will be trapped and suspended. The acidic nitrogen oxides of combustlon will react with the suspension to give a useful nitrogenous fertilizer slurry. Instead of generating an unpleasant smoky exhaust, the new engine will return its waste usefully to the land.

Thus modern wasteful agriculture will be reformed. Even old-style agriculture was inefficient, in the sense that the greater part of any crop plant cannot be eaten by men or animals. But Daedalus's scheme uses all the inedible and waste material. Instead of being rejected by dumping or burning, these biological wastes will be used as fuel, and their mineral contents recovered as fertilizer. Even modern industrial farming waste, such as plastic bags and old tyres, will be reclaimed. 